\title{
An Arcsine Law for Markov Random Walks
}

\author{
Gerold Alsmeyer and Fabian Buckmann
}

\begin{abstract}
The classic arcsine law for the number $N_{n}^{>}:=n^{-1} \sum_{k=1}^{n} \mathbf{1}_{\left\{S_{k}>0\right\}}$ of positive terms, as $n \rightarrow \infty$, in an ordinary random walk $\left(S_{n}\right)_{n \geq 0}$ is extended to the case when this random walk is governed by a positive recurrent Markov chain $\left(M_{n}\right)_{n \geq 0}$ on a countable state space $\mathcal{S}$, that is, for a Markov random walk $\left(M_{n}, S_{n}\right)_{n \geq 0}$ with positive recurrent discrete driving chain. More precisely, it is shown that $n^{-1} N_{n}^{>}$converges in distribution to a generalized arcsine law with parameter $\rho \in[0,1]$ (the classic arcsine law if $\rho=1 / 2$ ) iff the Spitzer condition

$$
\lim _{n \rightarrow \infty} \frac{1}{n} \sum_{k=1}^{n} \mathbb{P}_{i}\left(S_{n}>0\right)=\rho
$$

holds true for some and then all $i \in \mathcal{S}$, where $\mathbb{P}_{i}:=\mathbb{P}\left(\cdot \mid M_{0}=i\right)$ for $i \in \mathcal{S}$. It is also proved, under an extra assumption on the driving chain if $0<\rho<1$, that this condition is equivalent to the stronger variant

$$
\lim _{n \rightarrow \infty} \mathbb{P}_{i}\left(S_{n}>0\right)=\rho .
$$

For an ordinary random walk, this was shown by Doney [7 for $0<\rho<1$ and by Bertoin and Doney [3] for $\rho \in\{0,1\}$.
\end{abstract}

AMS 2000 subject classifications: 60J15 (60J10, 60G50)

Keywords: Markov random walk, arcsine law, fluctuation theory, Spitzer condition, Spitzertype formula

\section{Introduction}

The purpose of this note is to provide an arcsine law for the average number of positive sums $S_{k}=\sum_{l=1}^{n} X_{l}$ up to time $n$ as $n \rightarrow \infty$ when the increments $X_{1}, X_{2}, \ldots$ are modulated or driven by a positive recurrent Markov chain $\boldsymbol{M}=\left(M_{n}\right)_{n \geq 0}$ with countable state space $\mathcal{S}$. More precisely, the $X_{n}$ are conditionally independent given $\boldsymbol{M}$, and

Inst. Math. Stochastics, Department of Mathematics and Computer Science, University of Münster, Orléans-Ring 10, D-48149 Münster, Germany.

e-mail: gerolda@math.uni-muenster.de, f_buck01@uni-muenster.de 


$$
\mathbb{P}\left(\left(X_{1}, \ldots, X_{n}\right) \in \cdot \mid M_{0}=i_{0}, \ldots, M_{n}=i_{n}\right)=K_{i_{0} i_{1}} \otimes \ldots \otimes K_{i_{n-1} i_{n}}
$$

for all $n \geq 1, i_{0}, \ldots, i_{n} \in \mathcal{S}$ and some stochastic kernel $K$ from $\mathcal{S}^{2}$ to $\mathbb{R}$. Then $\left(M_{n}, S_{n}\right)_{n \geq 0}$, and sometimes also its additive part $\left(S_{n}\right)_{n \geq 0}$, is called a Markov random walk (MRW) or Markov additive process and $\boldsymbol{M}$ its driving chain. Let $P=\left(p_{i j}\right)_{i, j \in \mathcal{S}}$ denote the transition matrix of $M$ and $\pi=\left(\pi_{i}\right)_{i \in \mathcal{S}}$ its unique stationary distribution. For any $i \in \mathcal{S}$, we put further $\mathbb{P}_{i}:=\mathbb{P}\left(\cdot \mid M_{0}=i\right), \mathbb{P}_{\pi}:=\sum_{i \in \mathcal{S}} \pi_{i} \mathbb{P}_{i}$ and denote by $\left(\tau_{n}(i)\right)_{n \geq 1}$ the renewal sequence of successive return epochs of $\boldsymbol{M}$ to $i$.

If there exists a measurable function $g: \mathcal{S} \rightarrow \mathbb{R}$ such that $K_{i j}=\delta_{g(j)-g(i)}$, thus $X_{n}=$ $g\left(M_{n}\right)-g\left(M_{n-1}\right)$ a.s. for all $n \in \mathbb{N}$, then the MRW is called null-homologous, a term coined by Lalley [14], and it is called nontrivial otherwise. Here "a.s." means $\mathbb{P}_{i}$-a.s. for all $i \in \mathcal{S}$.

In [1, a wide range of fluctuation-theoretic results for $\left(M_{n}, S_{n}\right)_{n \geq 0}$ has been established by the natural approach of drawing on corresponding results for the embedded ordinary ran-

dom walks $\left(S_{\tau_{n}(i)}\right)_{n \geq 1}, i \in \mathcal{S}$, in combination with a thorough analysis of the excursions of the $S_{n}$ between the successive visits of the driving chain to a state $i$. Due to the fundamental observation that essential fluctuation-theoretic properties are shared by all embedded random walks (solidarity), the particular choice of $i$ does not matter for this approach. Here we will show that, if the limit

$$
\rho:=\lim _{n \rightarrow \infty} \frac{1}{n} \sum_{k=1}^{n} \mathbb{P}_{i}\left(S_{k}>0\right)
$$

exists for some $i \in \mathcal{S}$, then it does so and is the same for any $i \in \mathcal{S}$ (so we may replace $\mathbb{P}_{i}$ with $\mathbb{P}_{\pi}$ ), further satisfies

$$
\rho=\lim _{n \rightarrow \infty} \frac{1}{n} \sum_{k=1}^{n} \mathbb{P}_{i}\left(S_{\tau_{k}(i)}>0\right)
$$

for all $i \in \mathcal{S}$, and entails that an arcsine law holds for

$$
N_{n}^{>}:=\sum_{k=1}^{n} \mathbf{1}_{\left\{S_{k}>0\right\}} \quad\left(\text { and } \quad N_{n}^{\leqslant}:=n-N_{n}^{>}=\sum_{k=1}^{n} \mathbf{1}_{\left\{S_{k} \leq 0\right\}}\right) .
$$

The precise statement of the result is given as Theorem 1.1 below. Validity of (2) is known as Spitzer's condition for the ordinary zero-delayed random walk $\left(S_{\tau_{n}(i)}\right)_{n \geq 0}$ under $\mathbb{P}_{i}$, where $\tau_{0}(i):=0$, and is in fact equivalent to

$$
\rho=\lim _{n \rightarrow \infty} \mathbb{P}_{i}\left(S_{\tau_{n}(i)}>0\right),
$$

as shown by Doney [7] for $0<\rho<1$, and by Bertoin and Doney [3] for $\rho \in\{0,1\}$. Theorem 1.1 establishes also, under a second moment condition on $\tau(i)$ if $0<\rho<1$, the corresponding equivalence of (1) with

$$
\rho=\lim _{n \rightarrow \infty} \mathbb{P}_{i}\left(S_{n}>0\right)
$$

for any $i \in \mathcal{S}$.

Let $(A S(\theta))_{\theta \in[0,1]}$ be the family of generalized arcsine laws, i.e. $A S(0):=\delta_{0}, A S(1):=\delta_{1}$ and $A S(\theta)$ for $\theta \in(0,1)$ equals the beta distribution with parameters $1-\theta$ and $\theta$ and density 


$$
\frac{\sin (\pi \theta)}{\pi} \frac{1}{x^{1-\theta}(1-x)^{\theta}} \mathbf{1}_{(0,1)}(x) .
$$

For $\theta=\frac{1}{2}$, we get the classical arcsine law with distribution function

$$
A S(1 / 2)((-\infty, x])=\frac{2}{\pi} \arcsin (\sqrt{x}), \quad x \in[0,1] .
$$

The following arcsine law for nontrivial MRW generalizes the corresponding classical result for ordinary random walk due to Spitzer [18, Theorem 7.1], which in turn extended earlier versions by Lévy [15, Corollaire 2, p. 303] and Sparre Andersen [17, Theorem 3].

Theorem 1.1 (Arcsine law for MRWs) Let $\left(M_{n}, S_{n}\right)_{n \geq 0}$ be a nontrivial MRW with positive recurrent driving chain and consider the following assertions for arbitrary $i \in \mathcal{S}$ and $\rho \in[0,1]$ :

(a) Under $\mathbb{P}_{i}$,

$$
\frac{N_{n}^{>}}{n} \stackrel{d}{\rightarrow} A S(\rho) \quad \text { and } \quad \frac{N_{n}^{\leqslant}}{n} \stackrel{d}{\rightarrow} A S(1-\rho)
$$

as $n \rightarrow \infty$, where $\stackrel{d}{\rightarrow}$ means convergence in distribution.

(b) $\lim _{n \rightarrow \infty} n^{-1} \sum_{k=1}^{n} \mathbb{P}_{i}\left(S_{\tau_{k}(i)}>0\right)$ exists and equals $\rho$.

(c) Spitzer condition: $\lim _{n \rightarrow \infty} n^{-1} \sum_{k=1}^{n} \mathbb{P}_{i}\left(S_{k}>0\right)$ exists and equals $\rho$.

(d) Strong Spitzer condition: $\lim _{n \rightarrow \infty} \mathbb{P}_{i}\left(S_{n}>0\right)$ exists and equals $\rho$.

Then (a)-(c) are equivalent assertions and equivalence with (d) also holds true provided that $\mathbb{E}_{i} \tau(i)^{2}<\infty$ in the case $0<\rho<1$. Moreover, these assertions either hold for all $i \in \mathcal{S}$ with the same $\rho$ or none.

Remark 1.2 The previous result, more precisely its implication "(c) $\Rightarrow(a)$ ", was already shown by Freedman [9] for the special case when $X_{n}=g\left(M_{n}\right)$ for some measurable function $g$ and thus $S_{n}$ forms an additive functional of the driving chain. Regarding $g$, he further assumed $\mathbb{E}_{\pi}\left|X_{1}\right|=\sum_{i} \pi_{i}|g(i)|<\infty$, a condition not needed here.

Remark 1.3 In analogy to ordinary random walks, the classical arcsine law, that is (5) with $\rho=\frac{1}{2}$, is obtained if $\left(S_{n}\right)_{n \geq 0}$ satisfies a central limit theorem without centering, viz.

$$
\widehat{S}_{n}:=\frac{S_{n}}{n^{1 / 2}} \stackrel{d}{\rightarrow} \operatorname{Normal}\left(0, \theta^{2}\right)
$$

for some $\theta>0$. Namely, we then have

$$
\lim _{n \rightarrow \infty} \mathbb{P}\left(S_{n}>0\right)=\lim _{n \rightarrow \infty} \mathbb{P}\left(\widehat{S}_{n}>0\right)=\frac{1}{2}
$$

whence the assertion follows from part (d) of the above theorem. Note that $S_{n}$ may be viewed as an additive functional of the positive Harris chain $\left(M_{n}, X_{n}\right)_{n \geq 0}$. For such functionals, sufficient conditions for the validity of the central limit theorem, which typically include $\mathbb{E}_{\pi} X_{1}=0$ and $\mathbb{E}_{\pi} X_{1}^{2}<\infty$, have been studied by many authors, see e.g. Gordin and Lifšic [10], Woodroofe [19, Maxwell and Woodroofe [16, Derriennic and Lin [6] and also the references given therein. 
Let us further mention that, in view of Condition (b) and by similar reasoning as before, the classical arcsine law $A S(1 / 2)$ is also obtained if one (and by solidarity then all) of the ordinary embedded random walks $\left(S_{\tau_{n}(i)}\right)_{n \geq 0}$ satisfies the central limit theorem without centering, which is well-known to be true if $\mathbb{E}_{i} S_{\tau(i)}=\mathbb{E}_{\pi} X_{1} \mathbb{E}_{i} \tau(i)=0$ and $\mathbb{E}_{i} S_{\tau(i)}^{2}<\infty$, thus if $\left(S_{n}\right)_{n \geq 0}$ has stationary drift zero and finite variance over cycles determined by returns of the driving chain to a state $i$.

Remark 1.4 Albeit almost trivial, we note that $n^{-1} N_{n}^{>}$either converges in distribution to a generalized arcsine law $A S(\rho)$ or not at all. Namely, convergence to some law $Q$, say, on $[0,1]$ entails (by dominated convergence) that Theorem 1.1 (c) holds with $\rho:=\int x Q(d x)$ and thus $Q=A S(\rho)$ by Theorem 1.1(a).

Remark 1.5 Since $\left(S_{\tau_{n}(i)}\right)_{n \geq 0}$ is an ordinary random walk under $\mathbb{P}_{i}$, validity of Assertion (b) entails $\lim _{n \rightarrow \infty} n^{-1} \sum_{k=1}^{n} \mathbb{P}_{i}\left(S_{\tau_{k}(i)}<0\right)=1-\rho$ and thus validity of Theorem 1.1 for $\left(M_{n},-S_{n}\right)_{n \geq 0}$ as well (with $1-\rho$ instead of $\rho$ ). As a particular consequence, we infer that

$$
\lim _{n \rightarrow \infty} \frac{1}{n} \sum_{k=1}^{n} \mathbb{P}_{i}\left(S_{k}=0\right)=0
$$

for all $i \in \mathcal{S}$.

Remark 1.6 Let us further point out that Theorem 1.1(c) for all $i \in \mathcal{S}$ is also equivalent to

$$
\lim _{n \rightarrow \infty} \frac{1}{n} \sum_{k=1}^{n} \mathbb{P}_{\pi}\left(S_{k}>0\right)=\rho .
$$

While the necessity of (8) is obvious, the sufficiency proof needs a little more care and is deferred to Remark 2.7

Remark 1.7 Regarding the validity of the strong Spitzer condition (d), we do not know whether the additional assumption $\mathbb{E}_{i} \tau(i)^{2}<\infty$ in the case $0<\rho<1$ is really necessary but will provide an explanation in support of this in Remark 3.3 at the end of Subsection 3.1. On the other hand, the assumption is not very restrictive and particularly valid if the driving chain is geometrically ergodic or, a fortiori, has finite state space.

Remark 1.8 In the case of an ordinary random walk $\left(S_{n}\right)_{n \geq 0}$, two further arcsine laws, namely for

$$
L_{n}:=\min \left\{0 \leq k \leq n: S_{k}=\max _{0 \leq l \leq n} S_{l}\right\} \quad \text { and } \quad L_{n}^{\prime}:=\max \left\{0 \leq k \leq n: S_{k}=\min _{0 \leq l \leq n} S_{l}\right\}
$$

are directly derived by establishing $\left(N_{n}^{>}, S_{n}\right) \stackrel{d}{=}\left(L_{n}, S_{n}\right) \stackrel{d}{=}\left(L_{n}^{\prime}, S_{n}\right)$ for all $n \in \mathbb{N}_{0}$, where $\stackrel{d}{=}$ means equality in law. Since these distributional identities are no longer at hand in the Markov-modulated situation, arcsine laws for $L_{n}$ and $L_{n}^{\prime}$, if valid at all, require new arguments that will not be discussed here.

It is natural to expect, and confirmed by the next corollary, that the assertions of Theorem 1.1 if valid for $\left(M_{n}, S_{n}\right)_{n \geq 0}$, also hold for the dual MRW $\left({ }^{\#} M_{n},{ }^{\#} S_{n}\right)_{n \geq 0}$. Recall that, in the notation given above, the dual chain $\left(\# M_{n}\right)_{n \geq 0}$ has transition matrix ${ }^{\#} P=\left(\pi_{j} p_{j i} / \pi_{i}\right)_{i, j \in \mathcal{S}}$, while the conditional law of ${ }^{*} X_{n}={ }^{*} S_{n}-{ }^{*} S_{n-1}$ given ${ }^{*} M_{n-1}=i,{ }^{*} M_{n}=j$ equals 
${ }^{\#} K_{i j}=K_{j i}$ for all $i, j \in \mathcal{S}$. Since the embedded random walks $\left(S_{\tau_{n}(i)}\right)_{n \geq 0}$ and $\left({ }^{\#} S_{\#_{\tau_{n}}(i)}\right)_{n \geq 0}$ have the same distribution under $\mathbb{P}_{i}$ (w.l.o.g. put ${ }^{\#} M_{0}=M_{0}$ ), we see that Theorem [1.1(b), if valid for $\left(M_{n}, S_{n}\right)_{n \geq 0}$, also holds for the dual MRW. The announced corollary is now immediate.

Corollary 1.9 If, for some $\rho \in[0,1]$ and some/all $i \in \mathcal{S}$, the $M R W\left(M_{n}, S_{n}\right)_{n \geq 0}$ satisfies Theorem 1.1( (a)-(c), or (a)-(d), then the same holds true for its dual $\left({ }^{\#} M_{n},{ }^{\#} S_{n}\right)_{n \geq 0}$.

The further organization is as follows. The equivalence of Theorem 1.1(a)-(c) is established in the next section, while Section 3 deals with a proof of the strong Spitzer condition (d) if (a)-(c) hold. As a crucial ingredient, for the case $0<\rho<1$, we will there derive an extension of a Spitzer formula which may be of independent interest, see Proposition 3.1.

\section{Proof of Theorem 1.1(a)-(c)}

The proof of Theorem 1.1(a)-(c) (in fact, their equivalence) will be furnished by a number of auxiliary lemmata the first of which is cited from [2, Lemma 9.2] and particularly shows that any nontrivial ordinary random walk $\left(S_{n}\right)_{n \geq 0}$ converges to infinity in probability, a fact used in various places below.

Lemma 2.1 Let $\left(M_{n}, S_{n}\right)_{n \geq 0}$ be a nontrivial $M R W$ having positive recurrent driving chain $M=\left(M_{n}\right)_{n \geq 0}$ with stationary distribution $\pi$. Then $\left|S_{n}\right| \stackrel{\mathbb{P}_{\pi}}{\longrightarrow} \infty$, i.e.

$$
\lim _{n \rightarrow \infty} \mathbb{P}_{\pi}\left(\left|S_{n}\right| \leq x\right)=0
$$

for all $x>0$.

For the subsequent extension of Theorem 1.1(a), we put

$$
N_{n}^{>}(x):=\sum_{k=1}^{n} \mathbf{1}_{\left\{S_{k}>x\right\}} \quad \text { and } \quad N_{n}^{\leqslant}(x):=\sum_{k=1}^{n} \mathbf{1}_{\left\{S_{k} \leq x\right\}}
$$

for $n \in \mathbb{N}$ and $x \in \mathbb{R}$.

Lemma 2.2 Let $\left(M_{n}, S_{n}\right)_{n \geq 0}$ be a nontrivial $M R W$ with positive recurrent driving chain such that Theorem 1.1(a) holds for some $i \in \mathcal{S}$ and $\rho \in[0,1]$. Then under $\mathbb{P}_{i}$, as $n \rightarrow \infty$,

$$
\frac{N_{n}^{>}(x)}{n} \stackrel{d}{\rightarrow} A S(\rho) \quad \text { and } \quad \frac{N_{n}^{\leqslant}(x)}{n} \stackrel{d}{\rightarrow} A S(1-\rho)
$$

for all $x \in \mathbb{R}$.

Proof. Plainly, it is enough to prove the first assertion. Since $n^{-1} N_{n}^{>} \stackrel{d}{\rightarrow} A S(\rho)$, it suffices to note that (9) of Lemma 2.1 implies

$$
\frac{1}{n} \sum_{k=1}^{n} \mathbf{1}_{\left\{\left|S_{k}\right| \leq x\right\}} \stackrel{\mathbb{P}}{\rightarrow} 0
$$


for all $x \geq 0$ and that

$$
\frac{N_{n}^{>}}{n}-\frac{1}{n} \sum_{k=1}^{n} \mathbf{1}_{\left\{\left|S_{k}\right| \leq|x|\right\}} \leq \frac{N_{n}^{>}(x)}{n} \leq \frac{N_{n}^{>}}{n}+\frac{1}{n} \sum_{k=1}^{n} \mathbf{1}_{\left\{\left|S_{k}\right| \leq|x|\right\}}
$$

for all $x \in \mathbb{R}$.

A generalization of the classical arcsine law for ordinary random walks is next.

Lemma 2.3 Let $\left(X_{n}, Z_{n}\right)_{n \geq 1}$ be a sequence of i.i.d. bivariate random vectors such that $\mathbb{P}\left(X_{1}=0\right)<1$ and $\mathbb{E} Z_{1}=\mu \in(0, \infty)$. Define $S_{0}:=0$ and $S_{n}:=\sum_{k=1}^{n} X_{k}$ for $n \geq 1$. If $\left(S_{n}\right)_{n \geq 0}$ satisfies Spitzer's condition, i.e.

$$
\rho:=\lim _{n \rightarrow \infty} \frac{1}{n} \sum_{k=1}^{n} \mathbb{P}\left(S_{k}>0\right)
$$

exists, then

$$
\frac{1}{\mu n} \sum_{k=1}^{n} Z_{k} \mathbf{1}_{\left\{S_{k-1}>0\right\}} \stackrel{d}{\rightarrow} A S(\rho) \quad \text { and } \quad \frac{1}{\mu n} \sum_{k=1}^{n} Z_{k} \mathbf{1}_{\left\{S_{k-1} \leq 0\right\}} \stackrel{d}{\rightarrow} A S(1-\rho)
$$

as $n \rightarrow \infty$.

Proof. Since $\frac{1}{n} \sum_{k=1}^{n} Z_{k} \mathbf{1}_{\left\{S_{k-1} \leq 0\right\}}=\frac{1}{n} \sum_{k=1}^{n} Z_{k}-\frac{1}{n} \sum_{k=1}^{n} Z_{k} \mathbf{1}_{\left\{S_{k-1}>0\right\}}$, we see that the two assertions in (12) are equivalent and thus need to prove only the first one. We have $n^{-1} N_{n-1}^{>}(x) \stackrel{d}{\rightarrow} A S(\rho)$ by the classical arcsine law and

$$
\frac{1}{n} \sum_{k=1}^{n} Z_{k} \mathbf{1}_{\left\{S_{k-1}>0\right\}}=\mu \frac{N_{n-1}^{>}}{n}+\frac{1}{n} \sum_{k=1}^{n}\left(Z_{k}-\mu\right) \mathbf{1}_{\left\{S_{k-1}>0\right\}} .
$$

Hence it suffices to prove

$$
\frac{1}{n} \sum_{k=1}^{n}\left(Z_{k}-\mu\right) \mathbf{1}_{\left\{S_{k-1}>0\right\}} \stackrel{\mathbb{P}}{\rightarrow} 0
$$

as $n \rightarrow \infty$. But this follows directly from [12, Thm. 2.19] when observing that the sequence $\left(\sum_{k=1}^{n}\left(Z_{k}-\mu\right) \mathbf{1}_{\left\{S_{k-1}>0\right\}}\right)_{n \geq 0}$ forms a zero-mean martingale and

$$
\mathbb{P}\left(\left|Z_{k}-\mu\right|>z, S_{k-1}>0 \mid \mathcal{F}_{k-1}\right)=\mathbb{P}\left(\left|Z_{1}-\mu\right|>z\right) \mathbf{1}_{\left\{S_{k-1}>0\right\}} \leq \mathbb{P}\left(\left|Z_{1}-\mu\right|>z\right) \text { a.s. }
$$

for all $k \in \mathbb{N}$ and $z \geq 0$, where $\left(\mathcal{F}_{n}\right)_{n \geq 0}$ denotes the canonical filtration associated with $\left(X_{n}, Z_{n}\right)_{n \geq 1}$

For $n \in \mathbb{N}$ and $i \in \mathcal{S}$, we put

$$
\begin{aligned}
& D_{n}^{i}:=\max _{\tau_{n-1}(i)<k \leq \tau_{n}(i)}\left(S_{k}-S_{\tau_{n-1}(i)}\right)^{-}, \\
& H_{n}^{i}:=\max _{\tau_{n-1}(i)<k \leq \tau_{n}(i)}\left(S_{k}-S_{\tau_{n-1}(i)}\right)^{+}
\end{aligned}
$$

and 


$$
\chi_{n}(i):=\tau_{n}(i)-\tau_{n-1}(i)
$$

where $\tau_{0}(i):=0$. Obviously, the triplets $\left(D_{n}^{i}, H_{n}^{i}, \chi_{n}(i)\right)$ for $n \in \mathbb{N}$ are i.i.d. under $\mathbb{P}_{i}$.

Lemma 2.4 Let $\left(M_{n}, S_{n}\right)_{n \geq 0}$ be a nontrivial $M R W$ with positive recurrent driving chain such that Theorem $1.1(b)$ holds true for some $i \in \mathcal{S}$ and $\rho \in[0,1]$. Put

$$
L_{n}^{i,>}:=\frac{1}{n} \sum_{k=1}^{n} \chi_{k}(i) \mathbf{1}_{\left\{0<S_{\tau_{k-1}(i)} \leq D_{k}^{i}\right\}} \quad \text { and } \quad L_{n}^{i, \leqslant}:=\frac{1}{n} \sum_{k=1}^{n} \chi_{k}(i) \mathbf{1}_{\left\{-H_{k}^{i}<S_{\tau_{k-1}(i)} \leq 0\right\}}
$$

for $n \in \mathbb{N}$. Then $L_{n}^{i}:=L_{n}^{i,>}+L_{n}^{i, \leqslant}$ satisfies

$$
\lim _{n \rightarrow \infty} \mathbb{E}_{i} L_{n}^{i}=0
$$

in particular $L_{n}^{i} \stackrel{\mathbb{P}_{i}}{\rightarrow} 0$. Moreover,

$$
\frac{\pi_{i}}{n} \sum_{k=1}^{n} \chi_{k}(i) \mathbf{1}_{\left\{S_{\tau_{k-1}(i)}-D_{k}^{i}>0\right\}} \stackrel{d}{\rightarrow} A S(\rho)
$$

and

$$
\frac{\pi_{i}}{n} \sum_{k=1}^{n} \chi_{k}(i) \mathbf{1}_{\left\{S_{\tau_{k-1}(i)}+H_{k}^{i}>0\right\}} \stackrel{d}{\rightarrow} A S(\rho)
$$

under $\mathbb{P}_{i}$, as $n \rightarrow \infty$.

Proof. As noted before Lemma 2.1, $S_{\tau_{n}(i)} \stackrel{\mathbb{P}_{i}}{\longrightarrow} \infty$ and therefore

$$
\lim _{n \rightarrow \infty} \frac{1}{n} \sum_{k=1}^{n} \mathbb{P}\left(-y<S_{\tau_{k-1}(i)} \leq x\right)=0
$$

for all $x, y>0$. With the help of the dominated convergence theorem, this implies

$$
\begin{aligned}
& \lim _{n \rightarrow \infty} \mathbb{E}_{i} L_{n}^{i}=\lim _{n \rightarrow \infty} \frac{1}{n} \mathbb{E}_{i}\left(\sum_{k=1}^{n} \chi_{k}(i) \mathbf{1}_{\left\{-H_{k}^{i}<S_{\tau_{k-1}(i)} \leq D_{k}^{i}\right\}}\right) \\
& =\lim _{n \rightarrow \infty} \sum_{l \geq 1} \int \frac{1}{n} \sum_{k=1}^{n} \mathbb{P}\left(-y<S_{\tau_{k-1}(i)} \leq x\right) l \mathbb{P}_{i}\left(\tau(i)=l, D_{1}^{i} \in d x, H_{1}^{i} \in d y\right)=0,
\end{aligned}
$$

i.e. (13). Since $\pi_{i}=\left(\mathbb{E}_{i} \tau(i)\right)^{-1}$, we have by Lemma 2.3, when applied to the sequence $\left(S_{\tau_{n}(i)}-S_{\tau_{n-1}(i)}, \chi_{n}(i)\right)_{n \geq 1}$, that

$$
W_{n}:=\frac{\pi_{i}}{n} \sum_{k=1}^{n} \chi_{k}(i) \mathbf{1}_{\left\{S_{\tau_{k-1}(i)}>0\right\}} \stackrel{d}{\rightarrow} A S(\rho)
$$

under $\mathbb{P}_{i}$, as $n \rightarrow \infty$. Observing that 


$$
\frac{\pi_{i}}{n} \sum_{k=1}^{n} \chi_{k}(i) \mathbf{1}_{\left\{S_{\tau_{k-1}(i)}-D_{k}^{i}>0\right\}}=W_{n}-\pi_{i} L_{n}^{i,>}
$$

and

$$
\frac{\pi_{i}}{n} \sum_{k=1}^{n} \chi_{k}(i) \mathbf{1}_{\left\{S_{\tau_{k-1}(i)}+H_{k}^{i}>0\right\}}=W_{n}+\pi_{i} L_{n}^{i, \leqslant}
$$

the assertions (14) and (15) follow when combining $L_{n}^{i} \stackrel{\mathbb{P}_{i}}{\rightarrow} 0$ with (16).

Lemma 2.5 Let $\left(M_{n}, S_{n}\right)_{n \geq 0}$ be a nontrivial $M R W$ with positive recurrent driving chain such that Theorem 1.1(b) holds true for some $i \in \mathcal{S}$ and $\rho \in[0,1]$. Then

$$
\frac{N_{\tau_{n}(i)}^{>}}{\tau_{n}(i)} \stackrel{d}{\rightarrow} A S(\rho)
$$

under $\mathbb{P}_{i}$, as $n \rightarrow \infty$. Moreover, the same holds true when replacing $\tau_{n}(i)$ with $\tau_{\Lambda(n)}(i)$ or $\tau_{\Lambda(n)+1}(i)$, where $\Lambda(n):=\sup \left\{k \geq 0: \tau_{k}(i) \leq n\right\}$ for $n \in \mathbb{N}$.

Proof. We first point out that

$$
\left\{S_{\tau_{n-1}(i)}-D_{n}^{i}>0\right\} \subset\left\{S_{\tau_{n-1}(i)+k}>0\right\} \subset\left\{S_{\tau_{n-1}(i)}+H_{n}^{i}>0\right\}
$$

for all $n \in \mathbb{N}$ and $k=1, \ldots, \chi_{n}(i)$, hence

$$
\frac{1}{n} \sum_{k=1}^{n} \chi_{k}(i) \mathbf{1}_{\left\{S_{\tau_{k-1}(i)}-D_{n}^{i}>0\right\}} \leq \frac{N_{\tau_{n}(i)}^{>}}{n} \leq \frac{1}{n} \sum_{k=1}^{n} \chi_{k}(i) \mathbf{1}_{\left\{S_{\tau_{k-1}(i)}+H_{k}^{i}>0\right\}}
$$

for all $n \in \mathbb{N}$. Now use (13) in Lemma 2.4 to infer that the difference of the upper and lower bound converges to 0 in $\mathbb{P}_{i}$-probability. Moreover, these bounds have the same asymptotic law by (14) and (15), giving $\frac{\pi_{i}}{n} N_{\tau_{n}(i)}^{>} \stackrel{d}{\rightarrow} A S(\rho)$ under $\mathbb{P}_{i}$. Since $n^{-1} \tau_{n}(i) \rightarrow \pi_{i}^{-1} \mathbb{P}_{i}$-a.s. by the strong law of large numbers, Slutsky's theorem implies (17).

Replacing $\tau_{n}(i)$ with $\tau_{\Lambda(n)}(i)$ or $\tau_{\Lambda(n)+1}(i)$, the same result is obtained by an appeal to Anscombe's theorem [11, p. 16] because

$$
\frac{\tau_{\Lambda(n)}}{n} \stackrel{n \rightarrow \infty}{\longrightarrow} 1 \quad \mathbb{P}_{i \text {-a.s. entails }} \quad \frac{\Lambda(n)}{n}=\frac{\Lambda(n)}{\tau_{\Lambda(n)}} \cdot \frac{\tau_{\Lambda(n)}}{n} \stackrel{n \rightarrow \infty}{\longrightarrow} \frac{1}{\mathbb{E}_{i} \tau(i)}=\pi_{i} \quad \mathbb{P}_{i} \text {-a.s. }
$$

and

$$
\forall \varepsilon, \eta>0: \exists \delta>0, n_{0} \in \mathbb{N}: \forall n \geq n_{0}: \mathbb{P}_{i}\left(\max _{m:|m-n|<n \delta}\left|\frac{N_{\tau_{m}(i)}^{>}}{\tau_{m}(i)}-\frac{N_{\tau_{n}(i)}^{>}}{\tau_{n}(i)}\right|>\varepsilon\right)<\eta
$$

as one can readily check.

Lemma 2.6 Let $\left(M_{n}, S_{n}\right)_{n \geq 0}$ be a nontrivial $M R W$ with positive recurrent driving chain such that Theorem 1.1 (c) holds true for some $i \in \mathcal{S}$ and $\rho \in[0,1]$. Then Theorem 1.1 (b) for the same $i$ and $\rho$ is also valid.

Proof. Keeping the notation from the previous lemma, notice that $\Lambda(n) \leq n$ and recall that $n^{-1} \Lambda(n) \rightarrow \pi_{i} \mathbb{P}_{i}$-a.s. As a consequence, $\Lambda^{\prime}(n):=\Lambda(n) \vee n_{\varepsilon}$ and $\Lambda^{\prime \prime}(n):=\Lambda(n) \wedge n^{\varepsilon}$, 
where $n_{\varepsilon}:=\left\lceil(1-\varepsilon) \pi_{i} n\right\rceil$ and $n^{\varepsilon}:=\left\lceil(1+\varepsilon) \pi_{i} n\right\rceil$ for any fixed $\varepsilon \in(0,1)$, satisfy $\Lambda^{\prime}(n)-$ $\Lambda(n) \rightarrow 0$ and $\Lambda(n)-\Lambda^{\prime \prime}(n) \rightarrow 0 \mathbb{P}_{i}$-a.s. Moreover, for any stopping time $\nu$ for the sequence $\left(\tau_{k}(i), S_{\tau_{k}(i)}\right)_{k \geq 0}$, the identity

$$
\begin{aligned}
\mathbb{E}_{i}\left(\sum_{k=1}^{\nu} \chi_{k}(i)\right. & \left.\mathbf{1}_{\left\{S_{\tau_{k-1}(i)}>0\right\}}\right)=\sum_{k \geq 1} \mathbb{E}_{i} \chi_{k}(i) \mathbf{1}_{\left\{S_{\tau_{k-1}(i)}>0, \nu>k-1\right\}} \\
= & \mathbb{E}_{i} \tau(i) \sum_{k \geq 1} \mathbb{P}_{i}\left(S_{\tau_{k-1}(i)}>0, \nu>k-1\right) \\
& =\frac{1}{\pi_{i}} \mathbb{E}_{i}\left(\sum_{k=1}^{\nu} \mathbf{1}_{\left\{S_{\tau_{k-1}(i)}>0\right\}}\right)
\end{aligned}
$$

holds true and will be utilized hereafter for $\nu=\Lambda(n)+1=\inf \left\{k: \tau_{k}(i)>n\right\}$. Also noting that

$$
\begin{aligned}
\lim _{n \rightarrow \infty} \frac{1}{n} \mathbb{E}_{i} \chi_{\Lambda(n)+1}(i) & =0, \\
\lim _{n \rightarrow \infty} \frac{1}{n} \mathbb{E}_{i}\left(\sum_{k=1}^{\Lambda(n)+1} \chi_{k}(i) \mathbf{1}_{\left\{-H_{k}^{i}<S_{\tau_{k-1}(i)} \leq D_{k}^{i}\right\}}\right) & \leq \lim _{n \rightarrow \infty} \frac{n+1}{n} \mathbb{E}_{i} L_{n+1}^{i}=0,
\end{aligned}
$$

and

$$
\lim _{n \rightarrow \infty} \frac{1}{n} \mathbb{E}_{i}\left(\Lambda^{\prime}(n)-\Lambda(n)\right)=\lim _{n \rightarrow \infty} \frac{1}{n} \mathbb{E}_{i}\left(\Lambda^{\prime \prime}(n)-\Lambda(n)\right)=0,
$$

we now infer

$$
\begin{aligned}
\rho & =\lim _{n \rightarrow \infty} \frac{\mathbb{E}_{i} N_{n}^{>}}{n} \stackrel{\text { (20) }}{=} \lim _{n \rightarrow \infty} \frac{1}{n} \mathbb{E}_{i}\left(\sum_{k=1}^{\tau_{\Lambda(n)+1}(i)} \mathbf{1}_{\left\{S_{k}>0\right\}}\right) \\
& \leq \liminf _{n \rightarrow \infty} \frac{1}{n} \mathbb{E}_{i}\left(\sum_{k=1}^{\Lambda(n)+1} \chi_{k}(i) \mathbf{1}_{\left\{S_{\tau_{k-1}(i)}+H_{k}^{i}>0\right\}}\right) \\
& \stackrel{(21)}{=} \liminf _{n \rightarrow \infty} \frac{1}{n} \mathbb{E}_{i}\left(\sum_{k=1}^{\Lambda(n)+1} \chi_{k}(i) \mathbf{1}_{\left\{S_{\tau_{k-1}(i)}>0\right\}}\right) \\
& \stackrel{(19)}{=} \liminf _{n \rightarrow \infty} \frac{1}{\pi_{i} n} \mathbb{E}_{i}\left(\sum_{k=1}^{\Lambda(n)+1} \mathbf{1}_{\left\{S_{\tau_{k-1}(i)}>0\right\}}\right) \\
& \stackrel{(22)}{=} \liminf _{n \rightarrow \infty} \frac{1}{\pi_{i} n} \mathbb{E}_{i}\left(\sum_{k=1}^{\Lambda^{\prime \prime}(n)+1} \mathbf{1}_{\left\{S_{\tau_{k-1}(i)}>0\right\}}\right) \\
& \leq(1+\varepsilon) \liminf _{n \rightarrow \infty} \frac{1}{n^{\varepsilon}} \sum_{k=1}^{n^{\varepsilon}} \mathbb{P}_{i}\left(S_{\tau_{k-1}(i)}>0\right)
\end{aligned}
$$

and, conversely, 


$$
\begin{aligned}
& \rho \geq \limsup _{n \rightarrow \infty} \frac{1}{n} \mathbb{E}_{i}\left(\sum_{k=1}^{\Lambda(n)+1} \chi_{k}(i) \mathbf{1}_{\left\{S_{\tau_{k-1}(i)}-D_{k}^{i}>0\right\}}\right) \\
& \text { [21] } \limsup _{n \rightarrow \infty} \frac{1}{n} \mathbb{E}_{i}\left(\sum_{k=1}^{\Lambda(n)+1} \chi_{k}(i) \mathbf{1}_{\left\{S_{\tau_{k-1}(i)}>0\right\}}\right) \\
& \stackrel{\text { (19) }}{=} \limsup _{n \rightarrow \infty} \frac{1}{\pi_{i} n} \mathbb{E}_{i}\left(\sum_{k=1}^{\Lambda(n)+1} \mathbf{1}_{\left\{S_{\tau_{k-1}(i)}>0\right\}}\right) \\
& \stackrel{\text { (22) }}{=} \limsup _{n \rightarrow \infty} \frac{1}{\pi_{i} n} \mathbb{E}_{i}\left(\sum_{k=1}^{\Lambda^{\prime}(n)+1} \mathbf{1}_{\left\{S_{\tau_{k-1}(i)}>0\right\}}\right) \\
& \geq(1-\varepsilon) \limsup _{n \rightarrow \infty} \frac{1}{n_{\varepsilon}} \sum_{k=1}^{n_{\varepsilon}} \mathbb{P}_{i}\left(S_{\tau_{k-1}(i)}>0\right) \text {. }
\end{aligned}
$$

Since $\varepsilon \in(0,1)$ was arbitrarily chosen and $\left\{n_{\varepsilon}: n \in \mathbb{N}\right\}=\left\{n^{\varepsilon}: n \in \mathbb{N}\right\}=\mathbb{N}$ for all sufficiently small $\varepsilon$, we infer validity of Theorem 1.1(b).

Proof (of Theorem 1.1 (a)-(c)). Fix any $i \in \mathcal{S}$. Then (a) implies (c) by taking expectations, and (c) implies (b) by Lemma 2.6. To see that (b) implies (a), note first that Lemma 2.5 provides us with

$$
\frac{N_{\tau_{\Lambda(n)}(i)}^{>}}{\tau_{\Lambda(n)}(i)} \stackrel{d}{\rightarrow} A S(\rho) \text { and } \quad \frac{N_{\tau_{\Lambda(n)+1}(i)}^{>}}{\tau_{\Lambda(n)+1}(i)} \stackrel{d}{\rightarrow} A S(\rho)
$$

as $n \rightarrow \infty$. The assertion now follows because $N_{\tau_{\Lambda(n)}(i)}^{>} \leq N_{n}^{>} \leq N_{\tau_{\Lambda(n)+1}(i)}^{>}$and $\tau_{\Lambda(n)}(i) \leq$ $n \leq \tau_{\Lambda(n)+1}(i)$, thus

$$
\frac{N_{\tau_{\Lambda(n)}(i)}^{>}}{\tau_{\Lambda(n)+1}(i)} \leq \frac{N_{n}^{>}}{n} \leq \frac{N_{\tau_{\Lambda(n)+1}(i)}^{>}}{\tau_{\Lambda(n)}(i)}
$$

and $\tau_{\Lambda(n)+1}(i) / \tau_{\Lambda(n)}(i) \rightarrow 1 \mathbb{P}_{i \text {-a.s. }}$

In order to show that (a)-(c) hold under $\mathbb{P}_{j}$ for any $j \neq i$ as well, pick any $\varepsilon>0$ and an integer sequence $\left(k_{n}\right)_{n \geq 1}$ such that $k_{n} \rightarrow \infty$ and $n^{-1} k_{n} \rightarrow 0$. Fix $j \neq i$ and choose $x>0$ so large that $\mathbb{P}_{j}\left(\left|S_{\tau(i)}\right|>x\right)<\varepsilon$. Then

$$
\begin{aligned}
& \frac{1}{n} \sum_{k=1}^{n} \mathbb{P}_{j}\left(S_{k}>0\right) \leq \frac{1}{n} \mathbb{E}_{j}\left(\mathbf{1}_{\left\{\tau(i) \leq k_{n}\right\}} \sum_{k=\tau(i)}^{n} \mathbf{1}_{\left\{S_{k}>0\right\}}\right)+\frac{k_{n}}{n}+\mathbb{P}_{j}\left(\tau(i)>k_{n}\right) \\
& \leq \frac{1}{n} \mathbb{E}_{j}\left(\mathbf{1}_{\left\{\tau(i) \leq k_{n},\left|S_{\tau(i)}\right| \leq x\right\}} \sum_{k=\tau(i)}^{n} \mathbf{1}_{\left\{S_{k}>0\right\}}\right)+\frac{k_{n}}{n}+\mathbb{P}_{j}\left(\tau(i)>k_{n}\right)+\varepsilon \\
& \leq \frac{1}{n} \sum_{k=1}^{n} \mathbb{P}_{i}\left(S_{k}>-x\right)+\frac{k_{n}}{n}+\mathbb{P}_{j}\left(\tau(i)>k_{n}\right)+\varepsilon .
\end{aligned}
$$

Use Lemma 2.2 to see that 


$$
\lim _{n \rightarrow \infty} \frac{1}{n} \sum_{k=1}^{n} \mathbb{P}_{i}\left(S_{k}>y\right)=\lim _{n \rightarrow \infty} \frac{\mathbb{E}_{i} N_{n}^{>}(y)}{n}=\rho
$$

for all $y \in \mathbb{R}$. Therefore,

$$
\limsup _{n \rightarrow \infty} \frac{1}{n} \sum_{k=1}^{n} \mathbb{P}_{j}\left(S_{k}>0\right) \leq \rho+\varepsilon .
$$

By a similar argument, we find

$$
\begin{aligned}
\frac{1}{n} \sum_{k=1}^{n} \mathbb{P}_{j}\left(S_{k}>0\right) & \geq \frac{1}{n} \mathbb{E}_{j}\left(\mathbf{1}_{\left\{\tau(i) \leq k_{n},\left|S_{\tau(i)}\right| \leq x\right\}} \sum_{k=\tau(i)}^{n} \mathbf{1}_{\left\{S_{k}>0\right\}}\right) \\
& \geq \mathbb{P}_{j}\left(\tau(i) \leq k_{n},\left|S_{\tau(i)}\right| \leq x\right)\left(\frac{1}{n} \sum_{k=1}^{n-k_{n}} \mathbb{P}_{i}\left(S_{k}>x\right)\right) \\
& \geq\left(1-\mathbb{P}_{j}\left(\tau(i)>k_{n}\right)-\varepsilon\right)\left(\frac{1}{n} \sum_{k=1}^{n} \mathbb{P}_{i}\left(S_{k}>x\right)-\frac{k_{n}}{n}\right)
\end{aligned}
$$

and thereby

$$
\liminf _{n \rightarrow \infty} \frac{1}{n} \sum_{k=1}^{n} \mathbb{P}_{j}\left(S_{k}>0\right) \geq(1-\varepsilon) \rho .
$$

Since $\varepsilon>0$ was arbitrary, we conclude validity of Theorem 1.1(c) for $j \neq i$ and thus also of (a) and (b) by the first part of the proof.

Remark 2.7 By adapting the previously given argument, it is now easily proved that (8) implies Theorem 1.1(c). First note that, by Lemma 2.1] we have

$$
\frac{1}{n} \sum_{k=1}^{n} \mathbb{P}_{\pi}\left(S_{k}>x\right)=\rho
$$

for all $x \in \mathbb{R}$. Fix $i \in \mathcal{S}$ and pick an arbitrary $\varepsilon \in(0,1)$. Choose $\left(k_{n}\right)_{n \geq 1}$ as above and $x$ such that $\mathbb{P}_{\pi}\left(\left|S_{\tau(i)}\right| \leq x\right)<\varepsilon$. Then

$$
\frac{1}{n} \sum_{k=1}^{n} \mathbb{P}_{\pi}\left(S_{k}>x\right) \leq \frac{1}{n} \sum_{k=1}^{n} \mathbb{P}_{i}\left(S_{k}>0\right)+\frac{k_{n}}{n}+\mathbb{P}_{j}\left(\tau(i)>k_{n}\right)+\varepsilon
$$

and

$$
\frac{1}{n} \sum_{k=1}^{n} \mathbb{P}_{\pi}\left(S_{k}>-x\right) \geq\left(1-\mathbb{P}_{\pi}\left(\tau(i)>k_{n}\right)-\varepsilon\right)\left(\frac{1}{n} \sum_{k=1}^{n} \mathbb{P}_{i}\left(S_{k}>0\right)-\frac{k_{n}}{n}\right)
$$

and from this one easily concludes Theorem 1.1(c) for the chosen $i \in \mathcal{S}$. 


\section{The strong Spitzer condition: Proof of Theorem 1.1(d)}

\subsection{The case $0<\rho<1$}

For an ordinary random walk $\left(S_{n}\right)_{n \geq 0}$, Doney's [7] proof of the equivalence of the Spitzer condition and its strong version is based on the Spitzer-type formula (see [8, Eq. (7.7) on p. 414])

$$
\mathbb{P}\left(S_{n}>0\right)=\sum_{k \geq 1} \frac{n}{k} \mathbb{P}(\sigma(k)=n)
$$

where $\sigma(k)$ denotes the (possibly defective) $k$-th strictly ascending ladder epoch of $\left(S_{n}\right)_{n \geq 0}$. The subsequent proposition provides a substitute for this formula in the Markov-modulated situation which again uses Spitzer's combinatorial argument but for the i.i.d. blocks defined by the successive returns of the driving chain to an arbitrarily fixed state.

Proposition 3.1 Let $\left(M_{n}, S_{n}\right)_{n \geq 0}$ be a $M R W$ with positive recurrent driving chain on $\mathcal{S}$. For any fixed $i \in \mathcal{S}$, let $(\sigma(n))_{n \geq 1}$ be the (possibly defective) sequence of strictly ascending ladder epochs of the embedded random walk $\left(S_{\tau_{n}(i)}\right)_{n \geq 0}$ under $\mathbb{P}_{i}$. Then

$$
\mathbb{P}_{i}\left(M_{n}=i, S_{n}>0\right)=\sum_{k \geq 1} \frac{n}{k} \mathbb{E}_{i}\left(\frac{\sigma(k)}{\tau_{\sigma(k)}(i)} \mathbf{1}_{\left\{\tau_{\sigma(k)}(i)=n\right\}}\right) .
$$

for all $n \in \mathbb{N}$.

Notice that (24) reduces to (23) as it must if $\left(M_{n}\right)_{n \geq 0}$ is a single-state Markov chain and thus $\left(S_{n}\right)_{n \geq 0}$ an ordinary random walk.

Proof. For fixed $m, n \in \mathbb{N}$, consider the event $A_{m, n}:=\left\{\tau_{m}(i)=n, S_{n}>0\right\}$ and note that $\mathbf{1}_{A_{m, n}}=\mathbf{1}_{B_{m, n}}\left(Y_{1}, \ldots, Y_{m}\right)$, where

$$
B_{m, n}:=\left\{\left(i_{j}, x_{j}\right)_{1 \leq j \leq n} \in(\mathcal{S} \times \mathbb{R})^{n}: i_{n}=i, \sum_{r=1}^{n} \mathbf{1}_{\{i\}}\left(i_{r}\right)=m \text { and } \sum_{r=1}^{n} x_{r}>0\right\}
$$

and

$$
Y_{l}:=\left(M_{j}, X_{j}\right)_{\tau_{l-1}(i)+1 \leq j \leq \tau_{l}(i)}, \quad l \in \mathbb{N} .
$$

Put $\boldsymbol{Y}^{1}:=\left(Y_{1}, \ldots, Y_{m}\right), \boldsymbol{Y}^{2}:=\left(Y_{m}, Y_{1}, \ldots, Y_{m-1}\right), \ldots, \boldsymbol{Y}^{m}:=\left(Y_{2}, \ldots, Y_{m}, Y_{1}\right)$, which are the cyclic rearrangements of the i.i.d. block vectors $Y_{1}, \ldots, Y_{m}$ and thus identically distributed (under $\mathbb{P}_{i}$ ). Denote by

$$
\boldsymbol{S}^{l}=\left(S_{1}^{l}, \ldots, S_{n}^{l}\right), \quad l=1, \ldots, m
$$

the resulting vectors of partial sums after the rearrangements, thus $\boldsymbol{S}^{1}:=\left(S_{1}, \ldots, S_{n}\right)$. Notice that $\mathbf{1}_{B_{m, n}}\left(\boldsymbol{Y}^{1}\right)=\ldots=\mathbf{1}_{B_{m, n}}\left(\boldsymbol{Y}^{m}\right)$ and $S_{n}^{l}=S_{n}$ for each $l=1, \ldots, m$.

Now fix any $k \in \mathbb{N}$ and suppose that $k$ is the number of strict record values among those $S_{j}$ in $S^{1}$ with $M_{j}=i$, in other words, the number of strictly ascending ladder heights in $\left\{S_{\tau_{1}(i)}, \ldots, S_{\tau_{m-1}(i)}, S_{\tau_{m}(i)}\right\}$, i.e. $\sigma(k) \leq m<\sigma(k+1)$. We can write this event as $\left(\boldsymbol{S}^{1}\right)^{-1}\left(B_{k, m, n}\right)$ for some $B_{k, m, n} \subset B_{m, n}$. The crucial fact to be used hereafter is that the number $k$ does not vary for the vectors $\boldsymbol{S}^{l}$, thus $\left(\boldsymbol{S}^{1}\right)^{-1}\left(B_{k, m, n}\right)=\ldots=\left(\boldsymbol{S}^{m}\right)^{-1}\left(B_{k, m, n}\right)$, 
and that $k$ is also the number of these vectors for which the terminal value $S_{n}^{l}$ is a record. This follows by a simple combinatorial argument (see [8, Lemma 1 on p. 412]). Defining $I_{k}^{l}:=1$ if $\boldsymbol{Y}^{l} \in B_{k, m, n}$ and $S_{n}^{l}$ is a record value, and $I_{k}^{l}:=0$ otherwise, it follows that $I_{k}^{1}+\ldots+I_{k}^{m}$ takes only the two values 0 and $k$. Since $I_{k}^{1}, \ldots, I_{k}^{m}$ are also identically distributed under $\mathbb{P}_{i}$ with

$$
\mathbb{E}_{i} I_{k}^{1}=\mathbb{P}_{i}\left(A_{m, n} \cap\{\sigma(k)=m\}\right)=\mathbb{P}_{i}\left(\sigma(k)=m, \tau_{\sigma(k)}(i)=n\right),
$$

we arrive at

$$
\mathbb{P}_{i}\left(\sigma(k)=m, \tau_{\sigma(k)}(i)=n\right)=\frac{1}{m} \mathbb{E}_{i}\left(I_{k}^{1}+\ldots+I_{k}^{m}\right)=\frac{k}{m} \mathbb{P}_{i}\left(I_{k}^{1}+\ldots+I_{k}^{m}=k\right) .
$$

On the other hand, the events $\left\{I_{k}^{1}+\ldots+I_{k}^{m}=k\right\}$ for $k \in \mathbb{N}$ are pairwise disjoint and their union is $A_{m, n}$, hence

$$
\begin{aligned}
\mathbb{P}_{i}\left(A_{m, n}\right) & =\sum_{k \geq 1} \frac{m}{k} \mathbb{P}_{i}\left(\sigma(k)=m, \tau_{\sigma(k)}(i)=n\right) \\
& =\sum_{k \geq 1} \mathbb{E}_{i}\left(\frac{\sigma(k)}{k} \mathbf{1}_{\left\{\sigma(k)=m, \tau_{\sigma(k)}(i)=n\right\}}\right) \\
& =\sum_{k \geq 1} \frac{n}{k} \mathbb{E}_{i}\left(\frac{\sigma(k)}{\tau_{\sigma(k)}(i)} \mathbf{1}_{\left\{\sigma(k)=m, \tau_{\sigma(k)}(i)=n\right\}}\right) .
\end{aligned}
$$

Now the assertion (24) follows upon summing both sides over $m \in \mathbb{N}$ and using that the left-hand side then equals $\mathbb{P}_{i}\left(M_{n}=i, S_{n}>0\right)$.

Formula (24) forms the key ingredient to the following lemma which in turn furnishes our proof of Theorem 1.1(d) in the case $0<\rho<1$.

Lemma 3.2 Let $\left(M_{n}, S_{n}\right)_{n \geq 0}$ be a MRW with positive recurrent driving chain satisfying $\mathbb{E}_{i} \tau(i)^{2}<\infty$ and Theorem $1.1(a)-(c)$ for some $\rho \in(0,1)$ and all $i \in \mathcal{S}$. Then

$$
\lim _{n \rightarrow \infty} \mathbb{P}_{i}\left(M_{n d}=i, S_{n d}>0\right)=d \rho \pi_{i}
$$

all $i \in \mathcal{S}$, where $d \in \mathbb{N}$ denotes the period of $\left(M_{n}\right)_{n \geq 0}$.

Proof. We may restrict ourselves to the case when $\left(M_{n}\right)_{n \geq 0}$ is aperiodic, thus $d=1$. Fix any $i \in \mathcal{S}$ and let $\mathbb{P}_{i}$ be the underlying probability measure. If Theorem 1.1 (b) holds, then $\sigma(1)$ lies in the domain of attraction of $\mathbf{S}(\rho)$, the one-sided stable law with index $\rho$ (see e.g. [4. Thm. 8.9.12]), and since $\tau_{\sigma(n)} / \sigma(n) \rightarrow \pi_{i}^{-1} \mathbb{P}_{i}$-a.s., the same holds true for $\tau_{\sigma(1)}$. In fact, we can choose a continuous increasing function $\vartheta:[0, \infty) \rightarrow[0, \infty)$ which has inverse $\vartheta^{-1}$ and is regularly varying with index $1 / \rho$ at $\infty$ such that $\tau_{\sigma(n)} / \vartheta(n)$ converges in distribution to $\mathrm{S}(\rho)$. Let $f$ denote its density. By making use of the local limit theorem of Gnedenko (see [13, Thm. 4.2.1]), Doney [7] showed that for all $\delta>0$

$$
\begin{aligned}
& \sum_{\vartheta^{-1}(\delta n) \leq k \leq \vartheta^{-1}(n / \delta)} \frac{n}{k \vartheta(k)} \mathbb{P}_{i}\left(\tau_{\sigma(k)}(i)=n\right) \\
& =\sum_{\vartheta^{-1}(\delta n) \leq k \leq \vartheta^{-1}(n / \delta)} \frac{n}{k \vartheta(k)} f\left(\frac{n}{\vartheta(k)}\right)+o(1)
\end{aligned}
$$




$$
=\rho \int_{\delta}^{1 / \delta} f(x) d x+o(1)
$$

as $n \rightarrow \infty$. Using this, we infer that, for any $\delta, \varepsilon>0$,

$$
\begin{aligned}
& \mathbb{P}_{i}\left(M_{n}=i, S_{n}>0\right) \\
& \geq \sum_{\vartheta^{-1}(\delta n) \leq k \leq \vartheta^{-1}(n / \delta)} \frac{n\left(\pi_{i}-\varepsilon\right)}{k} \mathbb{P}_{i}\left(\frac{\sigma(k)}{\tau_{\sigma(k)}(i)} \geq \pi_{i}-\varepsilon, \tau_{\sigma(k)}(i)=n\right) \\
& \geq\left(\pi_{i}-\varepsilon\right)\left[\sum_{\vartheta^{-1}(\delta n) \leq k \leq \vartheta^{-1}(n / \delta)} \frac{n}{k} \mathbb{P}_{i}\left(\tau_{\sigma(k)}(i)=n\right)-R_{n}(\delta)\right] \\
& =\left(\pi_{i}-\varepsilon\right)\left[\rho \int_{\delta}^{1 / \delta} f(x) d x-R_{n}(\delta)\right]+o(1)
\end{aligned}
$$

as $n \rightarrow \infty$, where

$$
\begin{aligned}
R_{n}(\delta):= & \sum_{\vartheta-1(\delta n) \leq k \leq \vartheta^{-1}(n / \delta)} \frac{n}{k} \mathbb{P}_{i}\left(\frac{\sigma(k)}{\tau_{\sigma(k)}(i)}<\pi_{i}-\varepsilon, \tau_{\sigma(k)}(i)=n\right) \\
\leq & \sum_{\vartheta^{-1}(\delta n) \leq k \leq \vartheta^{-1}(n / \delta)} \frac{n}{k} \mathbb{P}_{i}\left(\varepsilon<\frac{\sigma(k)}{\tau_{\sigma(k)}(i)}<\pi_{i}-\varepsilon, \tau_{\sigma(k)}(i)=n\right) \\
& +\sum_{\vartheta^{-1}(\delta n) \leq k \leq \vartheta^{-1}(n / \delta)} \frac{n}{k} \mathbb{P}_{i}\left(\sigma(k)<\varepsilon n, \tau_{\sigma(k)}(i)=n\right) .
\end{aligned}
$$

But for $\vartheta^{-1}(\delta n) \leq k \leq \vartheta^{-1}(n / \delta)$, we further find that

$$
\begin{aligned}
& \mathbb{P}_{i}\left(\varepsilon<\frac{\sigma(k)}{\tau_{\sigma(k)}(i)}<\pi_{i}-\varepsilon, \tau_{\sigma(k)}(i)=n\right) \\
& \quad \leq \mathbb{P}_{i}\left(\frac{\tau_{\sigma(k)}(i)-\pi_{i}^{-1} \sigma(k)}{\sigma(k)}>\frac{1}{\pi_{i}-\varepsilon}-\frac{1}{\pi_{i}}, \sigma(k)>\varepsilon n, \tau_{\sigma(k)}(i)=n\right) \\
& \leq \mathbb{P}_{i}\left(\sup _{m \geq \varepsilon n} \frac{\tau_{m}(i)-m \pi_{i}^{-1}}{m}>\frac{\varepsilon}{\pi_{i}\left(\pi_{i}-\varepsilon\right)}\right)
\end{aligned}
$$

and

$$
\mathbb{P}_{i}\left(\sigma(k)<\varepsilon n, \tau_{\sigma(k)}(i)=n\right) \leq \mathbb{P}_{i}\left(\frac{\tau_{\varepsilon n}(i)-\pi_{i}^{-1} \varepsilon n}{\varepsilon n}>\frac{1}{\varepsilon}-\frac{1}{\pi_{i}}\right)
$$

giving

$$
\begin{aligned}
R_{n}(\delta) & \leq \frac{n\left(\vartheta^{-1}(n / \delta)-\vartheta^{-1}(\delta n)\right)}{\vartheta^{-1}(\delta n)} \mathbb{P}_{i}\left(\sup _{m \geq \varepsilon n} \frac{\tau_{m}(i)-m \pi_{i}^{-1}}{m}>\varepsilon\right) \\
& \leq C n \mathbb{P}_{i}\left(\sup _{m \geq \varepsilon n} \frac{\tau_{m}(i)-m \pi_{i}^{-1}}{m}>\frac{\varepsilon}{\pi_{i}}\right)
\end{aligned}
$$


for some $C>0$ and any $\varepsilon>0$ sufficiently small (and with the convention that $\tau_{x}(i):=$ $\left.\tau_{\lceil x\rceil}(i)\right)$. But, for any $\varepsilon>0$,

$$
a_{n}:=n \mathbb{P}_{i}\left(\sup _{m \geq n} \frac{\tau_{m}(i)-m \pi_{i}^{-1}}{m}>\varepsilon\right) \stackrel{n \rightarrow \infty}{\longrightarrow} 0
$$

because $\mathbb{E}_{i} \tau(i)^{2}<\infty$ ensures $\sum_{n>1} n^{-1} a_{n}<\infty$, see Chow and Lai [5, Eq. (3.10) with $\alpha=1$ and $p=2$ ], and $a_{n} / n$ is nonincreasing. By combining the previous estimates and noting that $\int_{\delta}^{1 / \delta} f(x) d x \rightarrow 1$ as $\delta \rightarrow 0$, we conclude

$$
\liminf _{n \rightarrow \infty} \mathbb{P}_{i}\left(M_{n}=i, S_{n}>0\right) \geq \pi_{i} \rho .
$$

In view of Remark 1.5, we can repeat the argument for $\left(M_{n},-S_{n}\right)_{n \geq 0}$ to obtain

$$
\liminf _{n \rightarrow \infty} \mathbb{P}_{i}\left(M_{n}=i, S_{n}<0\right) \geq \pi_{i}(1-\rho)
$$

or, equivalently,

$$
\limsup _{n \rightarrow \infty} \mathbb{P}_{i}\left(M_{n}=i, S_{n} \geq 0\right) \leq \pi_{i} \rho .
$$

Finally, (25) follows by a combination of (26) and (27).

Proof (of Theorem 1.1 $(d)$ ). Assertion (d) is now easily derived as follows. Fix any $i \in \mathcal{S}$ and suppose first that the driving chain is aperiodic $(d=1)$. Then we obtain with the help of (25) and Lemma 2.1 that

$$
\begin{aligned}
\mathbb{P}_{i}\left(M_{n}=j, S_{n}>0\right) & =\sum_{k=1}^{n} \int \mathbb{P}_{j}\left(M_{n-k}=j, S_{n-k}>-x\right) \mathbb{P}_{i}\left(\tau(j)=k, S_{k} \in d x\right) \\
& =\sum_{k=1}^{\lfloor n / 2\rfloor} \int \mathbb{P}_{j}\left(M_{n-k}=j, S_{n-k}>0\right) \mathbb{P}_{i}\left(\tau(j)=k, S_{k} \in d x\right)+o(1) \\
& =\pi_{j} \rho+o(1)
\end{aligned}
$$

as $n \rightarrow \infty$ and thereupon $\lim _{n \rightarrow \infty} \mathbb{P}_{i}\left(S_{n}>0\right)=\rho$ by summation over $j$.

If $\left(M_{n}\right)_{n \geq 0}$ has period $d \geq 2$, then let $\mathcal{S}_{r}, r=0, \ldots, d-1$, denote the cyclic class of states that can be reached from $i$ at times $n d+r$ for $n \in \mathbb{N}_{0}$. For $j \in \mathcal{S}_{r}$, it then follows in a similar manner as before that

$$
\begin{aligned}
& \mathbb{P}_{i}\left(M_{n d+r}=j, S_{n d+r}>0\right) \\
& =\sum_{k=0}^{\lfloor n / 2\rfloor} \int \mathbb{P}_{j}\left(M_{(n-k) d}=j, S_{(n-k) d}>0\right) \mathbb{P}_{i}\left(\tau(j)=k d+r, S_{k d+r} \in d x\right)+o(1) \\
& =d \pi_{j} \rho+o(1)
\end{aligned}
$$

as $n \rightarrow \infty$ and thereupon, using $\pi\left(\mathcal{S}_{r}\right)=d^{-1}$,

$$
\mathbb{P}_{i}\left(S_{n d+r}>0\right)=\sum_{j \in \mathcal{S}_{r}} \mathbb{P}_{i}\left(M_{n d+r}=j, S_{n d+r}>0\right) \stackrel{n \rightarrow \infty}{\longrightarrow} \rho
$$


for each $r=0, \ldots, d-1$ which again proves Assertion (d).

Remark 3.3 Let us finally comment on the need for the extra condition $\mathbb{E}_{i} \tau(i)^{2}<\infty$ which we have used in the estimation of $R_{n}(\delta)$ for the conclusion that

$$
n_{\vartheta^{-1}(\delta n) \leq k \leq \vartheta(n / \delta)} \mathbb{P}_{i}\left(\frac{\sigma(k)}{\tau_{\sigma(k)}(i)}<\pi_{i}-\varepsilon, \tau_{\sigma(k)}(i)=n\right)=o(1)
$$

as $n \rightarrow \infty$. An approach more in line with Doney's argument in the i.i.d.-case would be to derive this from a local limit theorem for the pair $\left(\sigma(k)-\pi_{i} \tau_{\sigma(k)}, \tau_{\sigma(k)}\right)$. However, this would require some knowledge of the dependence structure between $\sigma(k)$ and $\tau_{\sigma(k)}$ so as to provide the right normalization of $\sigma(k)-\pi_{i} \tau_{\sigma(k)}$. We doubt that this is possible without any extra condition on the given MRW $\left(M_{n}, S_{n}\right)_{n \geq 0}$.

\subsection{The case $\rho \in\{0,1\}$}

It clearly suffices to consider the case $\rho=1$ for which we make use of the following result very similar to Lemma 1 by Bertoin and Doney [3] which actually goes back to Kesten as noted by them.

Lemma 3.4 Suppose that, for any fixed $i \in \mathcal{S}, \rho_{n}:=\mathbb{P}_{i}\left(S_{\tau_{n}(i)}>0\right) \rightarrow 1$ as $n \rightarrow \infty$. Then

$$
\lim _{n \rightarrow \infty} \mathbb{P}_{i}\left(S_{k}>0 \text { for } \tau_{2 n}(i) \leq k \leq \tau_{r n}(i)\right)=1
$$

for any fixed integer $r>2$.

Proof. We adapt the argument given by Bertoin and Doney [3, Lemma 1] and prove that

$$
\mathbb{P}_{i}\left(S_{k}>0 \text { for } \tau_{2 n}(i) \leq k \leq \tau_{r n}(i)\right) \geq(1-\delta)(1-\varepsilon)^{r} \rho_{n}^{r+1} .
$$

for any fixed integer $r>2$ and $\varepsilon \in(0,1)$, where $\delta=\delta(\varepsilon, n):=\left(1-\rho_{n}\right) / \varepsilon \rho_{n} \geq 0$. Obviously, this implies (28).

Fix any $\varepsilon \in(0,1)$, put $D_{n}:=\min _{0 \leq k \leq \tau_{n}(i)} S_{k}$ for $n \in \mathbb{N}$ and let $q_{n}$ be the conditional $\varepsilon$-quantile of $D_{n}$ given $S_{\tau_{n}(i)}>0$, thus $q_{n} \leq 0$ and

$$
\mathbb{P}_{i}\left(D_{n}<q_{n} \mid S_{\tau_{n}(i)}>0\right)<\varepsilon \leq \mathbb{P}_{i}\left(D_{n} \leq q_{n} \mid S_{\tau_{n}(i)}>0\right) .
$$

As a consequence,

$$
\mathbb{P}_{i}\left(D_{n} \leq q_{n}\right) \geq \varepsilon \rho_{n}
$$

Now put $\nu:=\inf \left\{k: D_{k} \leq q_{n}\right\}$. Then

$$
\begin{aligned}
\mathbb{P}_{i}\left(S_{\tau_{n}(i)} \leq 0\right) & \geq \mathbb{P}_{i}\left(S_{\tau_{n}(i)} \leq 0, D_{n} \leq q_{n}\right) \\
& \geq \sum_{k \leq n} \mathbb{P}_{i}(\nu=k) \mathbb{P}_{i}\left(S_{\tau_{n-k}(i)} \leq-q_{n}\right) \\
& \geq \mathbb{P}_{i}\left(D_{n} \leq q_{n}\right) \min _{0 \leq k \leq n} \mathbb{P}_{i}\left(S_{\tau_{k}(i)} \leq-q_{n}\right)
\end{aligned}
$$


which in combination with (30) gives

$$
\min _{0 \leq k \leq n} \mathbb{P}_{i}\left(S_{\tau_{k}(i)} \leq-q_{n}\right) \leq \frac{1-\rho_{n}}{\varepsilon \rho_{n}}=\delta
$$

and thus $\mathbb{P}_{i}\left(S_{\tau_{m}(i)}>-q_{n}\right) \geq 1-\delta$ for some integer $m=m(\varepsilon, n) \leq n$.

Finally, consider the event

$$
\begin{aligned}
& S_{\tau_{n}(i)}>0, S_{\tau_{n+m}(i)}-S_{\tau_{n}(i)}>-q_{n}, S_{\tau_{(s+1) n+m}(i)}-S_{\tau_{s n+m}(i)}>0, \\
& \quad \min _{0 \leq j \leq n}\left(S_{\tau_{s n+m}(i)+j}-S_{\tau_{s n+m}(i)}\right) \geq q_{n}, \quad s=1, \ldots, r
\end{aligned}
$$

on which we have $S_{k}>0$ for all $\tau_{n+m}(i) \leq k \leq \tau_{r n+m}(i)$. Since $m \leq n$, the asserted inequality (29) follows.

In order to prove Assertion (d) of Theorem 1.1 given that (a)-(c) hold, choose $m=m_{n}:=$ $\left\lceil\left(4 \pi_{i}\right)^{-1} n\right\rceil$ for $n \in \mathbb{N}$ and note that $n^{-1} \tau_{n}(i) \rightarrow \pi_{i}^{-1} \mathbb{P}_{i}$-a.s. implies

$$
\lim _{n \rightarrow \infty} \mathbb{P}_{i}\left(\tau_{2 m}(i)>n \text { or } \tau_{8 m}(i)<n\right)=0 .
$$

Since, furthermore,

$$
\mathbb{P}_{i}\left(S_{n}>0\right) \geq \mathbb{P}_{i}\left(S_{k}>0 \text { for } \tau_{2 m}(i) \leq k \leq \tau_{8 m}(i)\right)-\mathbb{P}_{i}\left(\tau_{2 m}(i)>n \text { or } \tau_{8 m}(i)<n\right),
$$

we finally infer with the help of (28)

$$
\liminf _{n \rightarrow \infty} \mathbb{P}_{i}\left(S_{n}>0\right)=1 .
$$

Acknowledgements We are most grateful to an anonymous referee for pointing out an error in an earlier version of this article. Both authors were partially supported by the Deutsche Forschungsgemeinschaft (SFB 878).

\section{References}

1. G. Alsmeyer and F. Buckmann. Fluctuation theory for Markov random walks. J. Theoret. Probab., 2017. To appear. Electronic version at https://dx.doi.org/10.1007/s10959-017-0778-9.

2. G. Alsmeyer and F. Buckmann. Stability of perpetuities in Markovian environment. J. Difference Equ. Appl., 23(4):699-740, 2017.

3. J. Bertoin and R. A. Doney. Spitzer's condition for random walks and Lévy processes. Ann. Inst. H. Poincaré Probab. Statist., 33(2):167-178, 1997.

4. N. H. Bingham, C. M. Goldie, and J. L. Teugels. Regular variation, volume 27 of Encyclopedia of Mathematics and its Applications. Cambridge University Press, Cambridge, 1989.

5. Y. S. Chow and T. L. Lai. Some one-sided theorems on the tail distribution of sample sums with applications to the last time and largest excess of boundary crossings. Trans. Amer. Math. Soc., 208:51-72, 1975.

6. Y. Derriennic and M. Lin. The central limit theorem for Markov chains with normal transition operators, started at a point. Probab. Theory Related Fields, 119(4):508-528, 2001.

7. R. A. Doney. Spitzer's condition and ladder variables in random walks. Probab. Theory Related Fields, 101(4):577-580, 1995. 
8. W. Feller. An Introduction to Probability Theory and Its Applications. Vol. II. $2^{\text {nd }}$ edn. John Wiley \& Sons Inc., New York, 1971.

9. D. A. Freedman. An arcsine law for Markov chains. Proc. Amer. Math. Soc., 14:680-684, 1963.

10. M. I. Gordin and B. A. Lifšic. Central limit theorem for stationary Markov processes. Dokl. Akad. Nauk SSSR, 239(4):766-767, 1978.

11. A. Gut. Stopped Random Walks. Limit Theorems and Applications. Springer Series in Operations Research and Financial Engineering. Springer, New York, $2^{\text {nd }}$ edition, 2009.

12. P. Hall and C. C. Heyde. Martingale Limit Theory and Its Application. Academic Press Inc. [Harcourt Brace Jovanovich Publishers], New York, 1980. Probability and Mathematical Statistics.

13. I. A. Ibragimov and Y. V. Linnik. Independent and Stationary Sequences of Random Variables. Wolters-Noordhoff Publishing, Groningen, 1971. With a supplementary chapter by I. A. Ibragimov and V. V. Petrov, Translation from the Russian edited by J. F. C. Kingman.

14. S. P. Lalley. Renewal theorem for a class of stationary sequences. Probab. Theory Relat. Fields, 72(2):195-213, 1986.

15. P. Lévy. Sur certains processus stochastiques homogènes. Compositio Math., 7:283-339, 1940.

16. M. Maxwell and M. Woodroofe. Central limit theorems for additive functionals of Markov chains. Ann. Probab., 28(2):713-724, 2000.

17. E. Sparre Andersen. On the fluctuations of sums of random variables. II. Math. Scand., 2:195-223, 1954.

18. F. Spitzer. A combinatorial lemma and its application to probability theory. Trans. Amer. Math. Soc., 82:323-339, 1956.

19. M. Woodroofe. A central limit theorem for functions of a Markov chain with applications to shifts. Stochastic Process. Appl., 41(1):33-44, 1992. 